\title{
Modeling, Analysis, Simulation and Control of Semiconductor Manufacturing Systems: A Generalized Stochastic Colored Timed Petri Net Approach
}

\author{
Ming-Hung Lin and Li-Chen Fu \\ Dept. of Computer Science and Information Engineering, \\ National Taiwan University, \\ Taipei, Taiwan, R.O.C \\ E-mail: lichen@ccms.ntu.edu.tw
}

\begin{abstract}
In this paper, a generalized stochastic colored timed Petri net(GSCTPN) is used to model an IC wafer fabrication system. There are two major sub-models: Process-Flow Model and Transportation Model. There are two different automated guided vehicle systems, namely, Interbay system and Intrabay system. For multiple-load and variable-speed AGV system, we embed a simple motion-planning rule and introduce a collision avoidance strategy in model to solve the variable speed and traffic jam problems of vehicles. The simple control policies for AGV's visiting and AGV's routing are discussed. The heuristic rules for lot release and lot scheduling are also studied. To obtain performance measures, simulation is used. To show the promising potential of the proposed work, a real-word IC wafer fabrication system is used as a target plant layout for implementation.
\end{abstract}

\section{Introduction}

The previous works in applying Petri nets to IC wafer fabrication are discussed below. Modeling, analysis, simulation, scheduling, and control of semiconductor manufacturing systems was performed by using Petri nets in [1]. It also serves as a good tutorial paper. Janneck [2] presents the modeling a die bonder with Petri nets. Xiong et al.[3] propose and evaluate two Petrinet based hybrid heuristic search strategies and their applications to semiconductor test facility scheduling. In [4], hybrid Petri nets are presented as tools for modeling and simulation of semiconductor manufacturing systems. The colored timed Petri net(CTPN) is used to model the furnace in IC wafer fabrication, based on CTPN, the dynamic behaviors of the furnace can be emulated [5]. Jeng et al. [6] report a project of applying Petri net methodologies to detailed modeling, qualitative analysis, and performance evaluation of the etching area in an IC wafer fabrication system located in Taiwan's Hsinchu Science Based Industrial Park.

In this paper, a generalized stochastic colored timed Petri net(GSCTPN) model is built to model the detailed behaviors of an IC wafer fabrication system. Some control policies generated based on that GSCTPN model are to optimize some a priori assigned performance criterion. The plant layout and process-

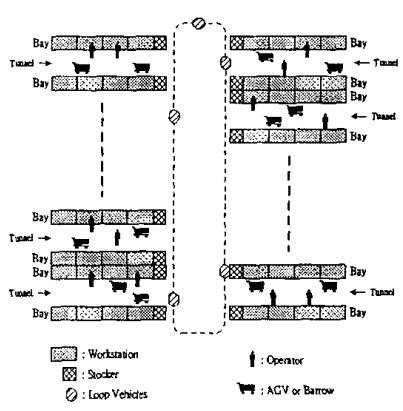

Figure 1: Physical Layout

ing steps are similar to that in $[1,6]$. Different from the model proposed in $[1,6]$, the present work introduces a GSCTPN modeling of a general IC wafer fabrication system. The result net model solves the "long" net modeling of reentrant processing problem and contains fewer of places and transitions; especially, including two different multiple-load and variable-speed AGV transportation systems. Although the AGV transportation system provides us flexibility and economy, it leaves us numerous ways of routing which unfortunately gives the control problem a combinatorial flavor. The organization of this paper is as follows. Section 2 describes the overview of IC manufacturing process and the IC plant layout. Section 3 introduces a GSCTPN Modeling of system. Section 4 describes a performance analysis based on simulation. Section 5 is conclusion.

\section{System Description}

In general, the machines in an IC wafer fabrication system are grouped into four functional sections: the photolithography area, the diffusion area, the etching area, and the thin film area. A processing unit of wafers is called a lot in an IC wafer fabrication. When lots arrive at the IC factory, they are processed in the previously mentioned four areas. As shown in Fig. 1, an IC plant is a base of tunnels. Machines are placed beside the tunnels, they are grouped into a bay. In the Interbay system, The corresponding AGV stops are arranged in location 25-35 as shown in Fig. 2. There is only one single-loop track in the Interbay system. In the In- 


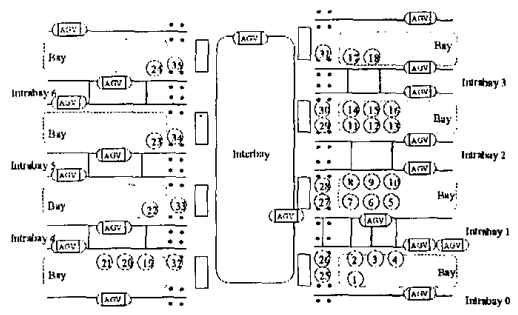

Figure 2: Plant Layout

terbay system, AGV that are called loop vehicles can move to any stop in unidirectional. Each stop in Interbay system is used as a transit station that transports lots between different bays. We denote the transit station as Interbay stationor stoker. There are 24 stations divided into 6 types, each of multi-server stations consists of several identical pieces of equipment. There are two types of lots associated with different wafer processing flows. One consists of 172 operations. The other consists of 148 operations.

\section{Generalized Stochastic Colored Timed Petri Net Modeling}

The proposed generalized stochastic colored timed Petri-Net (GSCTPN) extends the framework of the original PN by adding color, time and modular attributes to the net. The GSCTPN model contains two major sub-models. One is called Transportation Model, and the other is called Process-Flow Model.

\subsection{Modeling for Process Flow}

The Process Flow Model can be decomposed into two micro-models, each with different characteristics. One is Process Routing Module and the other is Process Elementary Module. The following are detailed descriptions of each of these micro-models.

\section{Process Routing Module}

The GSCTPN model we proposed to illustrate the processing flow of each lot type is Process Flow Model. It models the technological precedence constraints for processing lots and provides normally more than one alternative routes to accomplish the operation processing. The essential idea is that, the number of color set is equal to sum of adding total processing-steps for each type of lot. This means that the processing-steps attribute is embedded as colors to the model. For example, the number of processing steps for type 1 is 172 and that of type 2 is 148 . Then, the number of color is 320 . Token in color set $k$ represents that a lot is currently processing at the $k$-th step. Moreover, the number of tokens in color set $k$ represents the how many lots currently is at the $k$-th step.

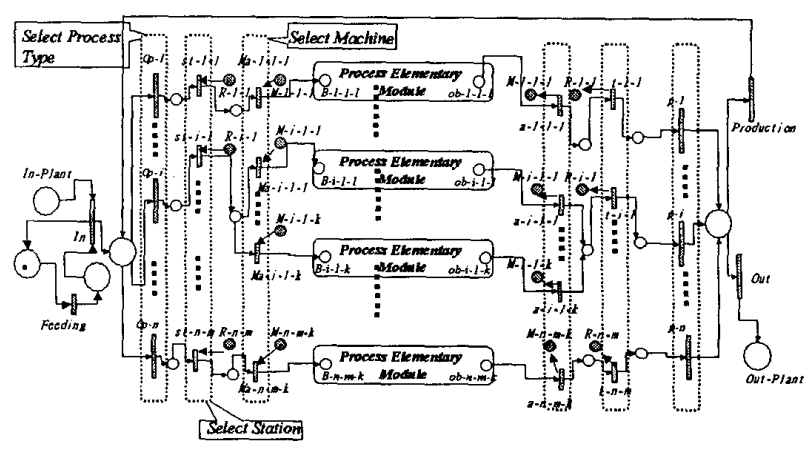

Figure 3: Process Routing Module

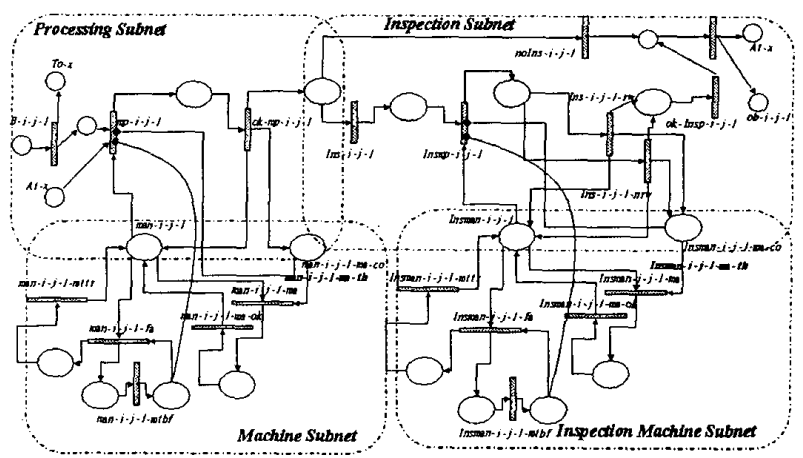

Figure 4: Process Elementary Module

\section{Process Elementary Module}

The Process Elementary Module can be composed of four subnets as shown in Fig. 4, each with different characteristics. They are, namely, Processing Subnet, Inspection Subnet, Machine Subnet, and Inspection Machine Subnet. However, the Inspection Subnet and Inspection Machine Subnet has to be additionally included if wafers need to be inspected. The following are detailed descriptions of each of these subnets.

The essential idea of modeling Processing Subnet is that, before a lot can take up any resource, it must acquire its control right in terms of a token. For example as shown in Fig. 4, if a lot wants to move to the machine Mac-i-j-l located at the stop $x$, it must get the token of resource place man- $i-j-l$. Moreover, if the current location where the wafer resides is not the same with that of machine Mac- $i-j-l$, it is need to send a signal in the communication place To- $x$. It invokes an AGV to come to the place where the wafer resides. When the AGV arrives, it proceeds to load the wafer. The AGV starts to move to the destination place.

Machines may fail, it is very common in IC fabrication. In Machine Subnet which is shown in Fig. 4, an operation transition man- $i-j-l$-mtbf will be fired periodically according to the fabrication statistics. It is called mean time between failure(MTBF). If transition 


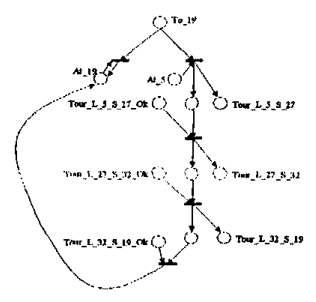

Figure 5: AGV-Calling Module

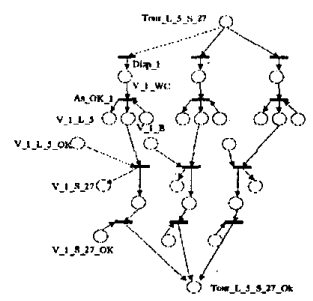

Figure 6: AGV-Calling Dispatching Module

man- $i-j-l-\mathrm{mtbf}$ is fired, intermediate transition man- $i-$ $j-l$-fa is enable and will be fired immediately. While man- $i-j-l-f a$ is fired, a token leaves from the resources place man- $i-j-l$. The repairing time is called mean time to repair(MTTR). While the time of operation transition man- $i-j-l$-mttr has passed, the machine repair has been completed and a token returns to place man- $i-j-l$.

\subsection{Modeling for Transportation System}

The Transportation Model can be decomposed into several micro-models, each with different characteristics. The following are detailed descriptions of each of these micro-models.

\section{AGV-Calling Module}

For example as shown in Fig. 5, a lot is located at the stop 5 currently and the next serving station is located at stop 19. Before the next operation, the communication place To-19 is marked to signal AGV and invokes an AGV to come to the place where the wafer resides. The stop 19 is located at different bay, it must be competed with the following steps. First, the communication place Tour_L_5_S_27 is marked. It means that wafer will have a tour loading from stop 5 and storing at stop 27. When the AGV arrives, AGV proceeds to load the wafer. Then, the AGV starts to move to the Interbay station 27 . A token in communication place Tour_L_5_S_27_ok indicates that the transporting tour from 5 to 27 is completed successfully.

\section{AGV-Calling Dispatching Module}

There are two considering in AGV-Calling Dispatching Module which is shown in Fig. 6. One is

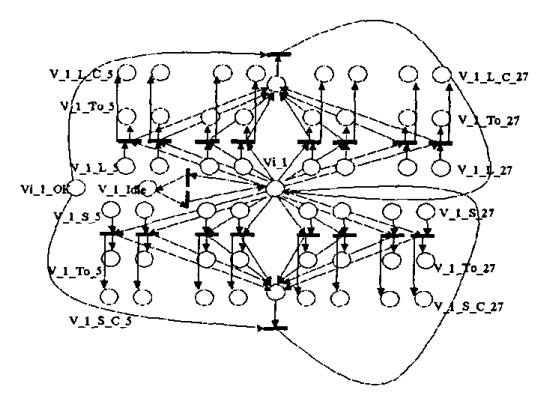

Figure 7: AGV-Visiting Planning Module

AGV-Calling Dispatching, the other is precedence constraints for transporting lots. When a token is in the communication place Tour_L_5_S_27, it indicates that a lot transporting to be served. It can be completed with two steps. First, an AGV is selected according to the dispatching policy. A good dispatching policy can make higher utilization of AGVs. Initially, we assume that there are three AGVs in the Intrabay 1. It is shown in Fig. 2. If AGV 1 is selected, the intermediate transition Disp.1 is fired. A token enters the intermediate place V_1_WC. It means that a lot is waiting for AGV 1 to transport. If the buffer of selected AGV is not full, the resource place V_1_B is marked. The intermediate transition As_OK_1 is enable and it can be fired.

\section{AGV-Visiting Planning Module}

Each multiple-load AGV may have to reach several stop points for loading or storing wafers. A good visiting planning is need to solve the traffic jam problems of vehicles. For example, there are eight stops $(2,3,4,5,6,7,26,27)$ in the Intrabay 1 . It is shown in Fig. 2. For example as shown in Fig. 7 , if the communication place V_1_L_5 is marked, it indicates that AGV 1 is need to arrive at stop 5 for loading a lot. Then, the communication place V_1_To_5 and V_1_L_C_5 are both marked. A token in the communication place V_1_To_5 represents that AGV 1 must visit the stop 5. A token in place V_1_L_C_5 represents that the goal of AGV 1 at stop 5 is to load a lot. If the communication place V_1_S_27 is marked, it represents that AGV 1 need to arrive at stop 27 for storing. A token in the communication place V_1_To_27 represents that AGV 1 must visit the stop 27. A token in place V_1_S_C_27 denotes that the goal of AGV 1 at stop 27 is to store a lot. Furthermore, when the communication place Vi_1_OK has a token, it means that the current visiting is finished and proceeds to next visiting. However, if there are no queues for visiting, the communication place V_1_Idle will be marked. It indicates that AGV 1 is at idle state. 


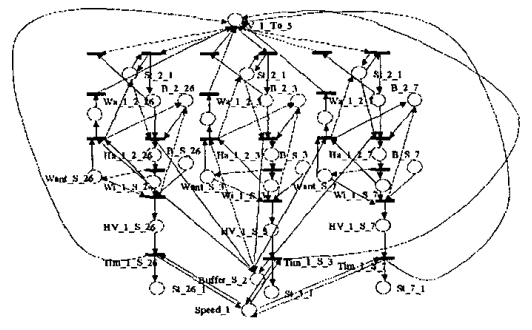

Figure 8: AGV Routing Module

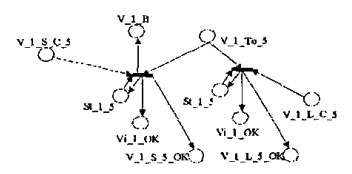

Figure 9: AGV Routing Module

\section{AGV Routing Module}

The basic concept of this model is described as follows. When an AGV needs to move from the current stop to the next adjacent stop, there are two steps to achieve that. Firstly, it needs to receive a 'right' of movement to the track between current stop and the next selected adjacent stop. If the 'right' is not ready, the selection of next adjacent stop can be performed again. If this condition is satisfied, it can start its traveling to that track. In Fig. 8, if AGV 1 selects adjacent stop 7 as its route, the intermediate place Wa_1_2_7 is marked. If the resource place $B \_2.7$ has tokens, it represents that the 'right' is ready. While the intermediate places Ha_1_2.7 is marked, tokens immediately enter both of the intermediate places Wi_1_S_7 and the communication place Want_S_7. It represents that AGV 1 is on the track going to stop 7 . At the same time, the control right of the stop 2 will be released to allow another AGV to use it as a destination or pass-by stop. A token in he communication place Want_S_7 represents that AGV 1 announces a 'right wanted' message to each of idle AGVs.

When AGV 1 arrives at stop 5 as shown in Fig. 9, one of two transitions will be fired according to the marking status of the communication places V_1_L_C_5 and V_1_S_C_5. A token will enter the communication place Vi_1_OK. It signals the AGV-Visiting Planning Module that current visiting is completed. Then, AGV proceeds to the next visiting. Either of the communication place V_1_S_5_OK and V_1_L_5_OK is marked. It represents that sending a signal to the AGV-Calling Dispatching Module to note that loading or storing at stop 5 is completed.

\section{AGV Idle Module}

The objective of the AGV Idle Module is again to introduce a control method into the Petri-Net model

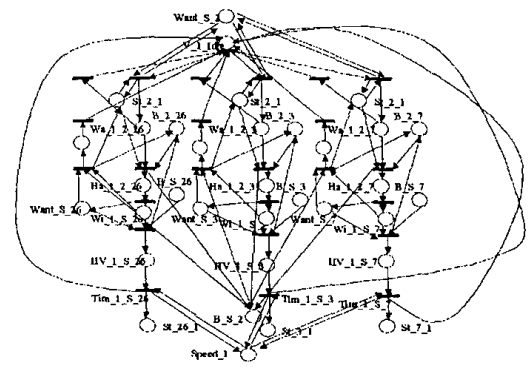

Figure 10: AGV Idle Module

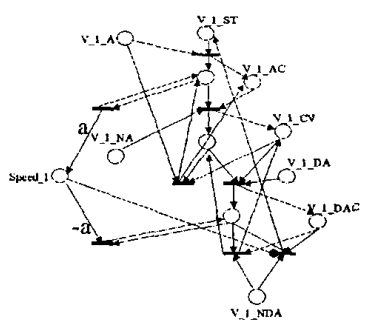

Figure 11: AGV Movement Module

with an aim to guaranteeing the jam-free conditions. The method we provide is a way called recursively leaving strategy.

\section{AGV Movement Module}

The purpose of the AGV Movement Module is to model the characteristics of AGV's movement. For example as shown in Fig. 11, the status of AGV 1's movement can be classified into four stages, namely, stop, accelerating, constant-speed and decelerating.

\section{AGV Motion Planning Module}

Each time when an AGV moves near from the next adjacent stop, it must be within two statuses. One is in non-braking status, the other is in braking status. For example, Fig. 12 illustrates the AGV Motion Planning Module, which guides an AGV to proper motion status while moving from the current stop 2. A token in the communication place V_1_Going represents that AGV 1 is in non-braking status. That the communication place V_1_Braking is marked means that it is in nonbraking status.

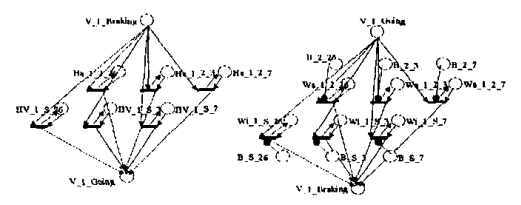

Figure 12: AGV Motion Planning Module 


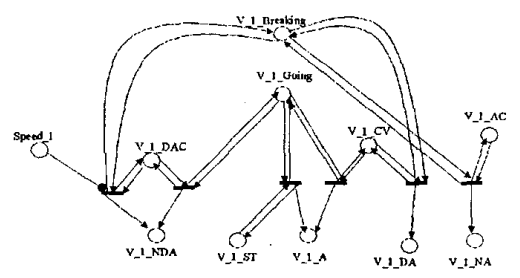

Figure 13: AGV Motion Control Module

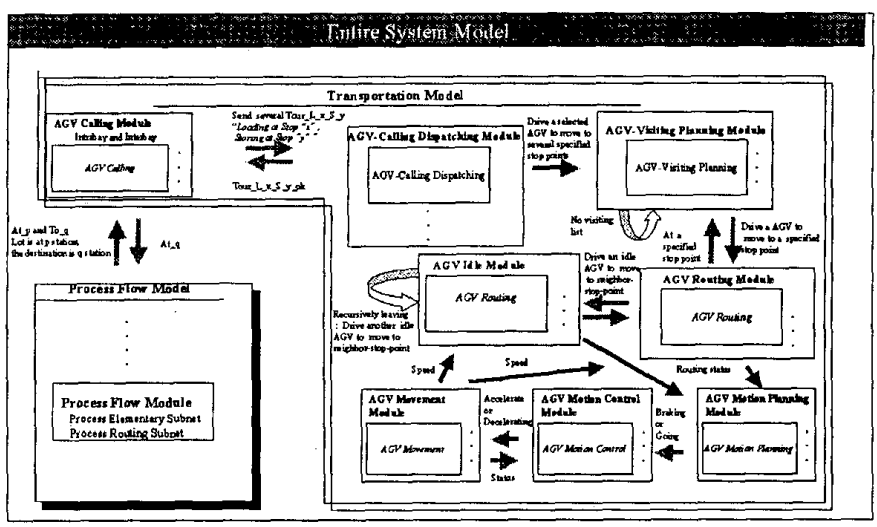

Figure 14: Model relationship graph

\section{AGV Motion Control Module}

The purpose of the AGV Motion Control Module is to embed a control rule into model so that AGV can take proper motion. The basic concept of this model is described as follows. When an AGV is in nonbreaking status and at 'stop' or 'constant-speed' stage mentioned in the AGV Movement Module, a 'start accelerating' command will be generated. It asks AGV to accelerate. For example, Fig. 13 illustrates the AGV Motion Control Module, which generate proper motion control commands for the AGV 1. A token in the communication place V_1_Going represents that AGV 1 is in non-braking status. That the communication place V.1_Braking is marked means that it is in non-braking status. The notations of other places and transitions are the same with those in AGV Movement Module.

\section{Control Strategies and Performance Analysis by Simulation}

Since the system model is large, simulation is used to obtain performance measures. In this simulation experiment, due to the nature of IC wafer processing, each operation time has little variation for different lots. A simulation experiment is conducted as follows.

\section{Lot Scheduling and Lot Release}

Firstly, let the release rule is DETERMIN and the ar-

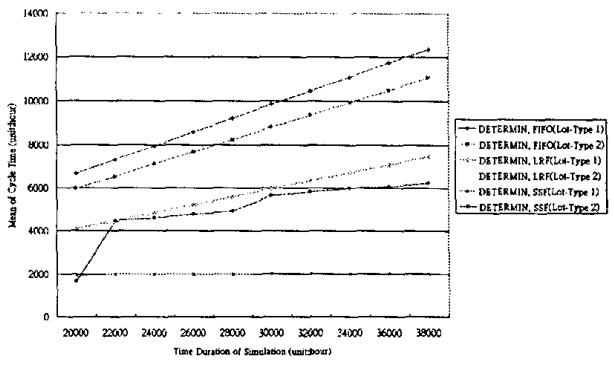

Figure 15: The relationship between lot scheduling rule and cycle time

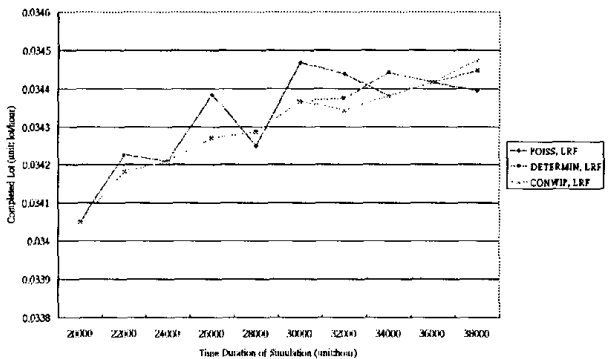

Figure 16: The relationship between lot release rule and number of completed lot

rival interval of input lot is 36 hours. It means that one lot is released into the wafer factory for every 36 hours. There are three scheduling rules, FIFO, LRF (Least remaining step firs), SSF(Shortest setup time first), to be implemented. Moreover, this simulation contains 10 runs. The time duration of the first run is 20000 hours and 38000 hours for the last run. The Fig. 15shows the simulation results, where SSF is better than the others do. We can see that the cycle time of lot is much long by using FIFO. Moreover, by using FIFO, the increasing rate of cycle time and WIP(work-in-process) is higher than the others.

We let the scheduling rule is LRF. The arrival interval of input lot is 36 hours for the constant input of DETERMIN(Inter-arrival times of lots are constant) and the mean of POISS(Lots enter the facility according to a Poisson process ). The WIP level keeps at 600 for CONWIP(Constant work-in-process). This simulation contains 10 runs. The time duration of the first run is 20000 hours and 38000 hours for the last run. In the Fig. 16, we can see that POISS having higher number of completed lots than DETERMIN and CONWIP at the initial runs with shorter period of time. When the time duration of run is large, CONWIP and DETERMIN are better than POISS. We also can see that the number of completed lots increases fast at the initial and arrives at the upper bound. 


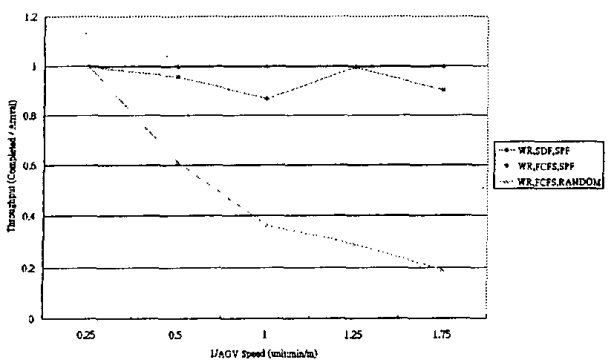

Figure 17: The relationship between speed of AGV and routing rule

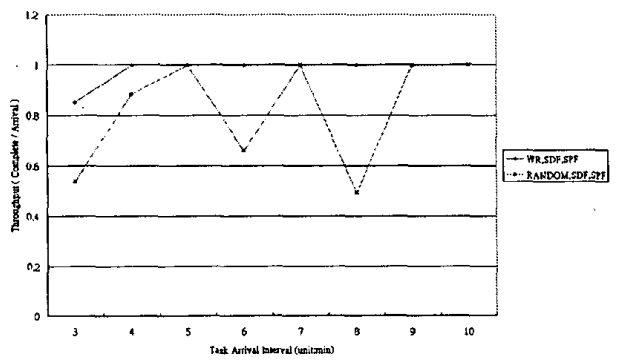

Figure 18: The relationship between visiting rule and task arrival interval

\section{AGV Routing and AGV Visiting}

In Intrabay 1, there are three multiple-load AGVs. There are three rule combinations. (WR(workload regulating), $\mathrm{SDF}$ (shortest distance first ), SPF(shortest path first )) denotes that the AGV dispatching rule is WR, visiting rule is SDF, and routing rule is SPF. (WR,FCFS,SPF) presents that the dispatching rule is WR, visiting rule is FCFS(fist come first serve), and routing rule is SPF. (WR,FCFS,RANDOM) presents that the dispatching rule is WR, visiting rule is FCFS(fist come first serve), and routing rule is RANDOM. Moreover, there are five runs in this simulation. The AGV's speed of the first run is $1 / 0.25 \mathrm{~m} / \mathrm{min}$ and $1 / 1.75 \mathrm{~m} / \mathrm{min}$ for the last run. In Fig. 17, we can see that the gap among three different combinations is increased according to $1 /$ (speed of AGV). This phenomenon indicates that increasing the speed of AGV can increase performance. The Fig. 17also show that the routing rule have more impact on performance than the visiting rule in this simulation experiment.

\section{AGV Dispatching}

There are two combinations,(WR,SDF,SPF),(RANDOM,SDF,SPF)tobe implemented. (WR,SDF,SPF) denotes that the AGV dispatching rule is $W R$, visiting rule is $\mathrm{SDF}$, and routing rule is SPF. (RANDOM,SDF,SPF) presents that the dispatching rule is RANDOM, visiting rule is SDF, and routing rule is SPF. The loading and storing tasks are fed into the Interbay 1. The task arrival is DETERMIN. Moreover, there are eight runs in this simulation. The task arrival interval of the first run is 3 minutes and that of the last is 10 minutes. The Fig. 18 show the results. The cycle time is increased according to the rate of task arrival. In this simulation experiment, dispatching rule RANDOM and WR are little difference in performance due to only three AGVs. If the number of AGVs is large, we can predict that RANDOM and WR are much difference in performance. The Fig. 18 also shows that WR have stable performance than RANDOM.

\section{CONCLUSION}

The present work introduces a GSCTPN modeling of a general IC wafer fabrication system, the result net model solves the "long" net modeling of reentrant processing problem. The validated model can be used to answer many "what-if" questions, such as predicting the throughput.

\section{REFERENCES}

[1] MengChu Zhou and Mu Der Jeng, "Modeling Analysis, Simulation, Scheduling, and Control of Semiconductor Manufacturing System: A petri Net Approach.", IEEE Tran. on Semiconductor Manufacturing, Vol. 11, pp. 333-357, 1998.

[2] Jorn W. Janneck and Martin Naedele, "Modeling a Die Bonder with Petri Nets : A Case Study.", IEEE Tran. on Semiconductor Manufacturing, Vol. 11, pp. 404-409, 1998.

[3] Huanxin Henry Xiong and MengChu Zhou, "Scheduling of SeMICONDUCTOR Test Facility via Petri Nets and Hybrid Heuristic Search.", IEEE Tran. on Semiconductor Manufacturing, Vol. 11, pp. 384-393, 1998.

[4] Mohamed Allam and Hassane Alla, "Modeling and Simulation of an Electronic Component Manufacturing System Using Hybrid Petri Nets.", IEEE Tran. on Semiconductor Manufacturing, Vol. 11, pp. 374-383, 1998.

[5] Sheng-ya Lin and Han-Pang Hung, "Modeling and Emulation of a Gurnace in IC Fab Based on Colored-Timed Petri Net.", IEEE Tran. on Semiconductor Manufacturing, Vol. 11, pp. 410-420, 1998.

[6] Mu Der Jeng, Xiaolan Xie and Shih Wei Chou, "Modeling, Qualitative Analysis, Simulation, and Performance Evaluation of the Etching Area in an IC Wafer Fabrication System Using Petri Nets.", IEEE Tran. on Semiconductor Manufacturing, Vol. 11, pp. 358-373, 1998. 